\title{
In vitro induction and in vivo engraftment of kidney organoids derived from human pluripotent stem cells
}

\author{
DENGLU ZHANG ${ }^{1,2}$, XIAOHANG DU ${ }^{2}$, XUFENG ZHANG ${ }^{2,3}$, KAILIN LI $^{2,4,5}$, \\ FENG KONG $^{2,4,5}$, GUANGHUI CHENG ${ }^{2,4,5}$ and SHENGTIAN ZHAO ${ }^{2,5-7}$
}

\author{
${ }^{1}$ Central Laboratory, Affiliated Hospital of Shandong University of Traditional Chinese Medicine, Jinan, \\ Shandong 250014; ${ }^{2}$ Key Laboratory for Kidney Regeneration of Shandong Province affiliated to The Second \\ Hospital of Shandong University; ${ }^{3}$ Department of Kidney Transplantation; ${ }^{4}$ Central Research Laboratory; ${ }^{5}$ Shandong \\ University-Karolinska Institute Collaborative Laboratory for Stem Cell Research; \\ ${ }^{6}$ Department of Urology, The Second Hospital, Shandong University, Jinan, Shandong 250033; ${ }^{7}$ Department of Urology, \\ Shandong Provincial Hospital Affiliated to Shandong University, Jinan, Shandong 250021, P.R. China
}

Received February 14, 2019; Accepted January 10, 2020

DOI: $10.3892 /$ etm.2020.8844

\begin{abstract}
The shortage of transplantable organs impedes the development of tissue-engineered alternatives. Producing tissues similar to immature kidneys from pluripotent stem cells is possible in vitro, but the size of the organoids is limited. Furthermore, in vivo implantation is necessary for organoid development and functional maturation. In the present study, the induction procedure was optimized and kidney organoids derived from induced pluripotent stem cells in vitro were produced. The kidney organoids were examined by immunofluorescence and quantitative PCR. Then, a unilateral nephrectomy model was established that was beneficial to the compensatory proliferation of the other kidney. Finally, these organoids were implanted below the kidney capsules of immunodeficient mouse hosts that had been nephrectomized unilaterally. This implantation resulted in the enlargement of the organoids and the production of vascular cells. Although signs of organoid maturation were lacking in short-term culture in vivo, the present study provided a method for studying kidney organoid development in vivo.
\end{abstract}

\section{Introduction}

Chronic kidney disease, especially end-stage renal disease, seriously threatens the life and health of humans (1). At present, blood dialysis and kidney transplantation are two

Correspondence to: Professor Shengtian Zhao, Department of Urology, Shandong Provincial Hospital Affiliated to Shandong University, 324 Jingwu Weiqi Road, Jinan, Shandong 250021, P.R. China

E-mail: zhaoshengtian@sdu.edu.cn

Key words: kidney, organoids, induced pluripotent stem cells, transplantation, medicine of the most commonly used strategies for the treatment of end-stage renal disease. Although blood dialysis can extend the life of patients, it can seriously influence their quality of life, and renal transplantation is considered the best method for treating end-stage renal disease $(2,3)$. At present, the kidneys used in transplantation originate from the donation of relatives or others. However, organ donation by itself cannot meet clinical needs, and cultivating functional and portable kidneys has substantial research significance and clinical value for resolving the kidney shortage problem (4).

A number of previous studies demonstrated that pluripotent stem cells could differentiate into mature cells and possess the potential to spontaneously assemble into tissues or organs $(5,6)$. In 2015, two research teams from Australia and the USA used different methods to induce human induced pluripotent stem cells (hiPSCs) into kidney organoids, comprising collecting tubes, proximal tubule cells, distal tubule cells, glomerular cells, endothelial cells and other cell types $(7,8)$. The kidney organoids did not possess kidney class structures but did have certain renal tubular absorption characteristics $(7,8)$.

However, inducing 3D renal structuress or organoids in vitro has two disadvantages; one is the nutrient supply problem, which limits the number of cells and thus also limits the number of nephrons that can be generated, and the other is the low degree of cell differentiation $(7,8)$. Previous experiments demonstrated that the stem cell differentiation microenvironment affects the induction of 3D renal structures or organoids and thus, could promote vascularization of the graft (9-11).

The renal capsule is one site that is often transplanted (9). As early as 2004, Hammerman (12) identified that renal primordia (metanephroi) transplanted into an animal renal capsule undergo organogenesis in situ, become vascularized by blood vessels of host origin and exhibit excretory function. Also, newly developed nephron/posterior renal primordia have been suggested to integrate into the host assembly system under the renal capsule thereby enhancing the renal function 
of the host (12). Recently, van den Berg et al (13) demonstrated that human pluripotent stem cell-derived kidney organoids, under the kidney capsule and in the absence of any exogenous vascular endothelial growth factor, develop host-derived vascularization. These previous studies demonstrated that the renal capsule is an ideal site for transplantation.

Unilateral nephrectomy can lead to compensatory hypertrophy of the other kidney, which is a common clinical phenomenon $(14,15)$. Taking this phenomenon into consideration, Dilworth et al (16) demonstrated that a left nephrectomy, which was performed on a host rat, improved the growth of transplanted metanephroi. Matsumoto et al (17) observed that grafts were well differentiated after the transplantation of fetal kidneys into the omenta or abdominal aortas of rats via unilateral nephrectomy. Therefore, a unilateral nephrectomy model may be beneficial for differentiation and vascularization of kidney organoids.

Therefore, the present study aimed to determine whether the microenvironment of the renal capsule in immunodeficient mice undergoing unilateral nephrectomy could promote vascularization and differentiation of kidney organoids. The present results may be of significance for renal regeneration research.

\section{Materials and methods}

hiPSCs. hiPSCs were gifted by Professor Zhiguo Chen (Cell Therapy Center, Beijing Institute of Geriatrics, Xuanwu Hospital Capital Medical University) (18) and were maintained in mTeSR1 medium (Stemcell Technologies, Inc.; cat. no. 85850) in 6-well cell culture plates (Costar; Corning, Inc.; cat. no. 3516) coated with $1 \%$ vol/vol human embryonic stem cell-qualified Matrigel (Corning, Inc.; cat. no. 354277 ) in a $37^{\circ} \mathrm{C}$ incubator at $5 \% \mathrm{CO}_{2}$. hiPSCs were passaged in Dissociation Solution for human embryonic stem cells/iPSCs (ReproCELL, Inc.; cat. no. RCHETP002) at a 1:3 ratio every 7 days, according to the manufacturer's protocol.

Optimizing the conditions of intermediate mesoderm formation in hiPSCs. hiPSCs were plated on a Matrigel-coated 6-well cell culture dish at 2,000 cells per $\mathrm{cm}^{2}$ in mTeSR1 medium. The next day, the cells had reached $20-30 \%$ confluence and were treated with gradient concentrations $(0,6$, 8, 10, 12 and $16 \mu \mathrm{M}$ ) of CHIR99021.e (Sigma-Aldrich; Merck KGaA; cat. no. SML1046) in APEL basal medium (Stemcell Technologies, Inc.; cat. no. 05270) supplemented with antibiotic-antimycotic (Thermo Fisher Scientific, Inc.; cat. no. 15240062) for 4 days .. The cells were then collected and the mRNA levels of T, TBX6 and LHX1 (molecular markers of primitive streaking) were detected by RT-qPCR. In the next step, the cells were treated with gradient concentrations $(0$, $100,150,200,250$ and $300 \mathrm{ng} / \mathrm{ml}$ ) of FGF9 (R\&D Systems, Inc.; cat. no. 273-F9/CF) for 3 days and $1 \mu \mathrm{g} / \mathrm{ml}$ heparin (Sigma-Aldrich; Merck KGaA; cat. no. 9041-08-1) was simultaneously added. On the 7th day, qPCR was used to detect the expression of the anterior intermediate mesoderm marker GATA3, and the posterior intermediate mesoderm markers HOXD11 and EYA1. The medium was changed every other day. The above induction processes were all in $37^{\circ} \mathrm{C}$ incubator.
The best concentration of inducer was selected by the expression of molecular marker.

$3 D$ kidney organoid formation. On day 7, the cells were collected and dissociated into single cells using trypsin or TrypLE select (Thermo Fisher Scientific, Inc.; cat. no. 1905777). Cells $\left(5 \times 10^{5}\right)$ were centrifuged at $400 \mathrm{x}$ g for $2 \mathrm{~min}$ at room temperature to form a pellet and then transferred onto a Transwell 0.4-mm-pore polyester membrane (Corning, Inc.; cat. no. CLS3450). The pellets were treated with $5 \mu \mathrm{M}$ CHIR99021 in APEL for $1 \mathrm{~h}$, cultured with FGF9 $(200 \mathrm{ng} / \mathrm{ml})$ and heparin $(1 \mu \mathrm{g} / \mathrm{ml})$ for 5 days, and cultured in APEL basal medium for another 2 weeks; the medium was changed three times a week. Culture medium was added to the lower chamber of the Transwell only and was in contact with the membrane but did not cross the membrane.

Immunofluorescence analysis of 3D organoids. 3D kidney organoids were fixed with $4 \%$ paraformaldehyde in PBS for $20 \mathrm{~min}$ at room temperature (RT) in a 96-well plate and then washed three times with PBS. The organoids were then incubated in blocking buffer $[0.3 \%$ Triton X-100 and 5\% normal donkey serum (cat. no. S9100; Beijing Solarbio Science and Technology Co., Ltd.) for $1 \mathrm{~h}$ at RT and washed three times with PBS. The organoids were incubated with primary antibodies in antibody dilution buffer $(0.3 \%$ Triton $\mathrm{X}-100$ and $1 \%$ BSA in PBS) overnight at $4^{\circ} \mathrm{C}$. The following antibodies and dilutions were used: Anti-Brachyury (1:500; cat. no. ab209665; Abcam), anti-LHX1 (1:500; cat. no. sc-515631; Santa Cruz Biotechnology, Inc.), anti-GATA3 (1:1,000; cat. no. ab199428; Abcam), anti-WT1 (1:500; cat. no. ab89901; Abcam), anti-ECAD (1:500; cat. no. ab1416; Abcam), anti-PODXL (1:500; cat. no. ab150358; Abcam), anti-NPHS1 (1:300; cat. no. sc-376522; Santa Cruz Biotechnology, Inc.), anti-CD31 (1:500; cat. no. ab24590; Abcam). The organoids were then washed with PBS three times for $1 \mathrm{~h}$ each. The 4 th wash was performed overnight at $4{ }^{\circ} \mathrm{C}$. For immunostaining with biotinylated Lotus tetragonolobus lectin (LTL; 1:300; cat. no. B-1325; Vector Laboratories, Inc.), a Streptavidin/Biotin Blocking kit (cat. no. SP-2002; Vector Laboratories, Inc.;) was used, according to the manufacturer's protocol. The organoids were incubated with secondary antibodies (1:500; Alexa Flour 488 labeled goat-anti-mouse IgG; cat.no. A0428; Beyotime Institute of Biotechnology or 1:500; cy3-labeled goat-anti-rabbit IgG; cat. no. A0516, Beyotime Institute of Biotechnology or 1:500; m-IgGk BP-PE; cat. no. SC-516141; Santa Cruz Biotechnology, Inc.) in antibody dilution buffer for $1 \mathrm{~h}$ at RT and then washed with PBS three times for $30 \mathrm{~min}$ each. Nuclei were counterstained with DAPI for more than $10 \mathrm{~min}$ at room temperature. The organoids were then mounted with Vectashield (Vector Laboratories, Inc.; cat. no. H-1200) and examined by confocal microscopy at x200 and x400 magnification (Zeiss AG; model no. LSM780).

Reverse transcription-quantitative PCR (RT-qPCR). Total RNA was purified from cells using the Total RNA kit I (Omega Bio-Tek, Inc.; cat. no. R6834-01). In total, 2 g RNA was used for RT with the ReverTra Ace qPCR RT kit (Toyobo Life Science; cat. no. 741200) according to the manufacturer's protocol. RT-qPCRs were conducted in duplicate 
using cDNA (1:100), $500 \mathrm{nM}$ forward and reverse primers and SYBR-Green (Toyobo Life Science; cat. no. 717000). RT-qPCR was performed using the Roche Real-Time PCR Detection System (Roche Diagnostics; LightCycler 480II). All samples were run in two technical replicates and GAPDH was used as the housekeeping gene. Values were calculated using the $2^{-\triangle \triangle C q}$ method (19). The following primers were used: GAPDH, forward, 5'-GGAGCGAGATCCCTCCAAAAT-3' and reverse, 5'-GGCTGTTGTCATACTTCTCATGG-3'; T, forward, 5'-CTGGGTACTCCCAATGGGG-3' and reverse, 5'-GGTTGGAGAATTGTTCCGATGA-3'; T-box transcription factor 6 (TBX6), forward, 5'-CATCCACGAGAATTG TACCCG-3' and reverse, 5'-AGCAATCCAGTTTAGGGG TGT-3'; LIM homeobox 1 (LHX1), forward, 5'-CCTGGA CCGCTTTCTCTTGAA-3' and reverse, 5'-ACCGAAACA CCGGAAGAAGTC-3'; GATA binding protein 3 (GATA3), forward, 5'-GCCCCTCATTAAGCCCAAG-3' and reverse, 5'-TTGTGGTGGTCTGACAGTTCG-3'; homeobox 11 (HOXD11), forward, 5'-TCGACCAGTTCTACGAGGCA-3' and reverse, 5'-AAAAACTCGCGTTCCAGTTCG-3'; EYA transcriptional coactivator and phosphatase 1 (EYA1), forward, 5'-GTCACAGTCTCAGTCACCTGG-3' and reverse, 5'-GGGATAAGACGGATAGTCCTGC-3'

Western blotting. Protein sample preparation and western blotting were performed as previously described (20). Blots were incubated with primary antibodies against PODXL (1:1,000; cat. no. ab150358; Abcam), RET (1:500; ab134100; Abcam) and WT1 (1:1,000; cat. no. ab89901; Abcam), and NPHS1 (1:500; cat. no. sc-376522; Santa Cruz Biotechnology, Inc.), WNT11 (1:500; cat. no. sc-365033; Santa Cruz Biotechnology, Inc.) and GAPDH (1:3,000; cat. no. sc-47724; Santa Cruz Biotechnology, Inc.) overnight at $4^{\circ} \mathrm{C}$, followed by appropriate peroxidase-conjugated secondary antibodies (1:3,000; anti-mouse IgG, HRP-linked; cat. no. 7076; Cell Signaling Technology, Inc.; anti-rabbit IgG, HRP-linked; cat. no. 7074; Cell Signaling Technology, Inc.). GAPDH served as an internal control. Visualization of the immunocomplexes was conducted using an enhanced chemiluminescent HRP substrate (cat. no. 1829501; EMD Millipore) and followed by exposure to X-ray films.

Animal surgery. Animal care and handling were performed in accordance with The National Institutes of Health Guide for the Care and Use of Laboratory Animals. All procedures were approved by The Ethics Committee of The Second Hospital of Shandong University. A total of 12 male immunodeficient mice (BAlB/c Nude; age, 5-6 weeks; weight, 22-25 g; Beijing Vital River Laboratory Animal Technology Company) were initially anesthetized with $1 \%$ pentobarbital sodium $(50 \mathrm{mg} / \mathrm{kg}$ ) via intraperitoneal injection. The right kidneys of the mice were removed and ligated with a surgical line. Mice were divided into a control group and an experimental group, with 6 mice in each group. In the experimental group, kidney organoids were transplanted into the kidney capsule, which were then sutured. In the control group, kidney organoids were transplanted into the subcutaneous armpit of mice. No mice died during the experiment. A total of 2 weeks after transplantation, all mice were euthanized with $1 \%$ pentobarbital sodium $(150 \mathrm{mg} / \mathrm{kg})$ via intraperitoneal injection. Criteria for judging death in mice included continuous no spontaneous breathing for 2-3 min and no blinking reflex.

The mice were maintained at $22-25^{\circ} \mathrm{C}$, at a relative humidity of 50-60\% and on a $12 \mathrm{~h}$ light/dark cycle. The health of the mice was observed every day and it was ensured that the mice had adequate access to food and water. The cages and mattresses were changed once per week and the mice were kept in a warm environment after anesthesia.

Hematoxylin and eosin staining. The graft was fixed in $4 \%$ paraformaldehyde for $48 \mathrm{~h}$ at room temperature and embedded in paraffin, which was then cut into sections with a thickness of $4 \mu \mathrm{m}$. An H\&E staining kit (cat. no. G1120; Beijing Solarbio Science \& Technology Co., Ltd.) was used according to the manufacturer's instructions. Images were captured using a light microscope at X100 magnification.

Statistical analysis. Statistical analysis was performed using GraphPad Prism software (version 5.0; GraphPad Software, Inc.). A paired Student's t-test was used for analysis of statistical significance between the control and treated groups. The comparative data are presented as the mean \pm SD of at least three independent experiments. $\mathrm{P}<0.05$ was considered to indicate a statistically significant difference.

\section{Results}

Optimizing the conditions of intermediate mesoderm formatoin in hiPSCs. The methods of inducing kidney organoid formation from hiPSCs have been widely discussed $(7,8)$. However, the dosages of factors required for induction of kidney organoid formation in different hiPSC strains may be different; therefore, the induction procedure was optimized according to the method by Takasato et al (7). First, hiPSCs were treated with gradient concentrations $(0,6,8,10,12$ and $16 \mu \mathrm{M}$ ) of CHIR99021 for 4 days in a $37^{\circ} \mathrm{C}$ incubator at $5 \% \mathrm{CO}_{2}$, before they were observed under a light microscope at x100 magnification (Fig. 1A). The cells were then collected and the mRNA levels of T, TBX6 and LHX1 (molecular markers of primitive streaking) were detected by RT-qPCR (Fig. 1B).

While $10 \mu \mathrm{M}$ CHIR99021 was determined to be the best concentration for induction based on the expression of TBX6, $12 \mu \mathrm{M}$ was deemed the best induction concentration based on the expression of $\mathrm{T}$ and LHX1. The present study used $12 \mu \mathrm{M}$ as the working concentration of CHIR99021. In the second step, the cells were treated with gradient concentrations of FGF9 $(0,100,150,200,250$ and $300 \mathrm{ng} / \mathrm{ml})$ for 3 days $(1 \mu \mathrm{g} / \mathrm{ml}$ heparin was simultaneously added). On the 7th day, qPCR was used to detect the expression of the anterior intermediate mesoderm marker GATA3, and the posterior intermediate mesoderm markers HOXD11 and EYA1 (Fig. 1C). Among these markers, the expression of HOXD11 increased with increasing FGF9 concentrations, while the expression of EYA1 was not affected by changes in the FGF9 concentration. Based on the expression of GATA3, $200 \mu \mathrm{g} / \mathrm{ml} \mathrm{FGF9}$ was determined to be the best concentration for induction.

During the induction, the cellular morphology was markedly altered. After the initial cell clones were treated with CHIR99021, they were dispersed and exhibited a long shuttle 
A

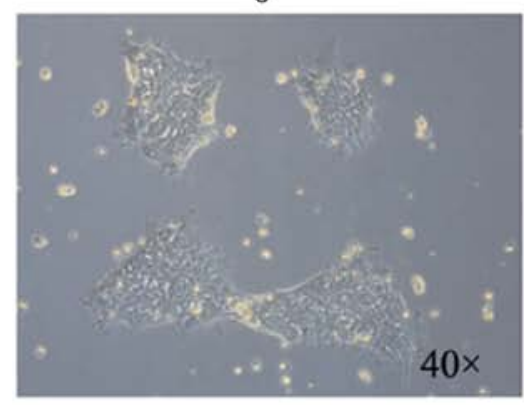

$10 \mu \mathrm{M}$

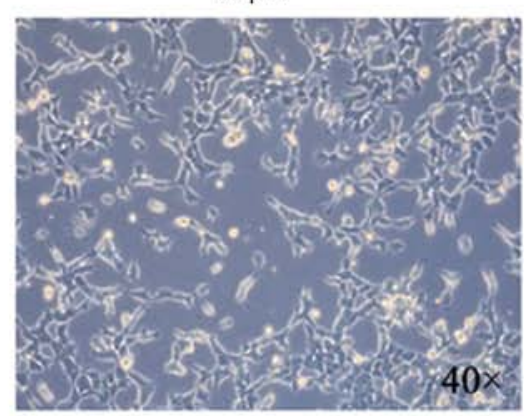

$6 \mu \mathrm{M}$

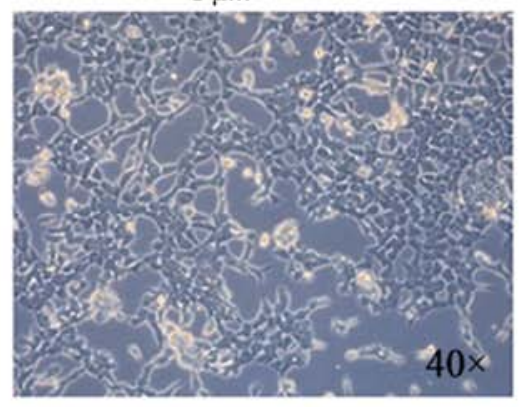

$12 \mu \mathrm{M}$

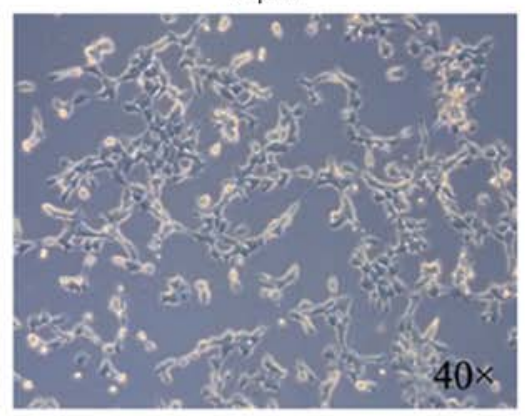

$8 \mu \mathrm{M}$
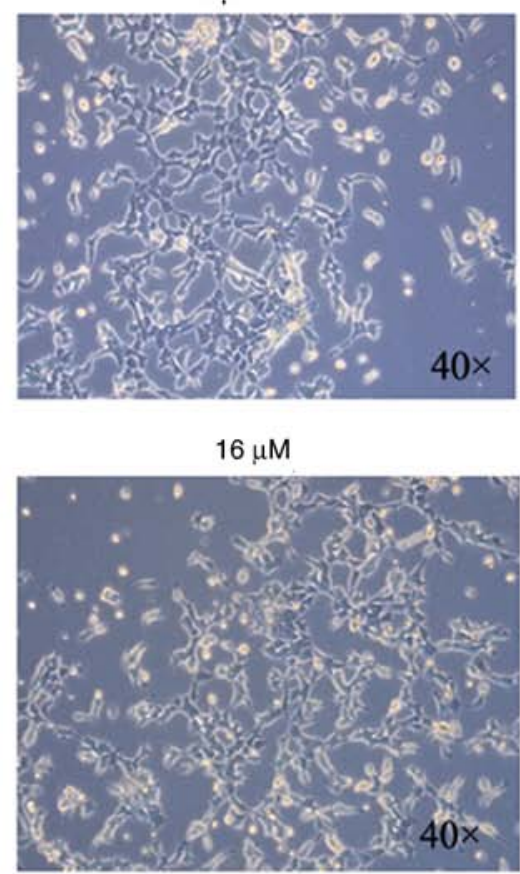

B

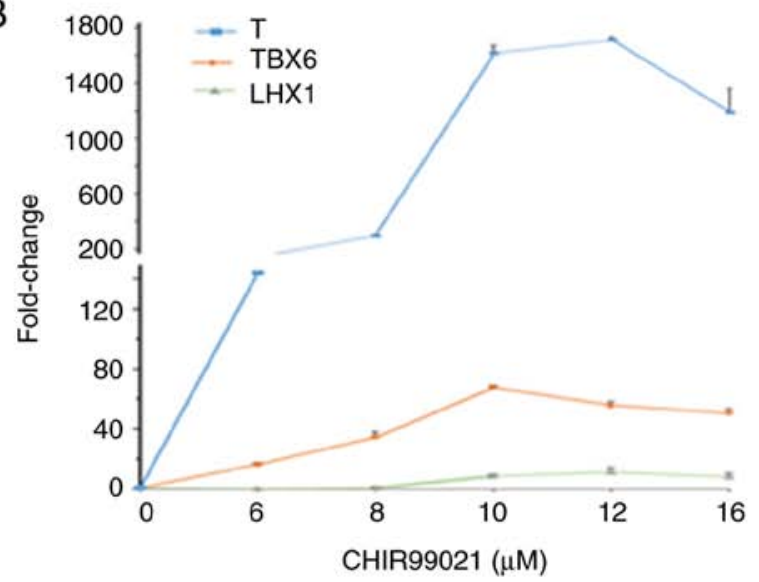

C

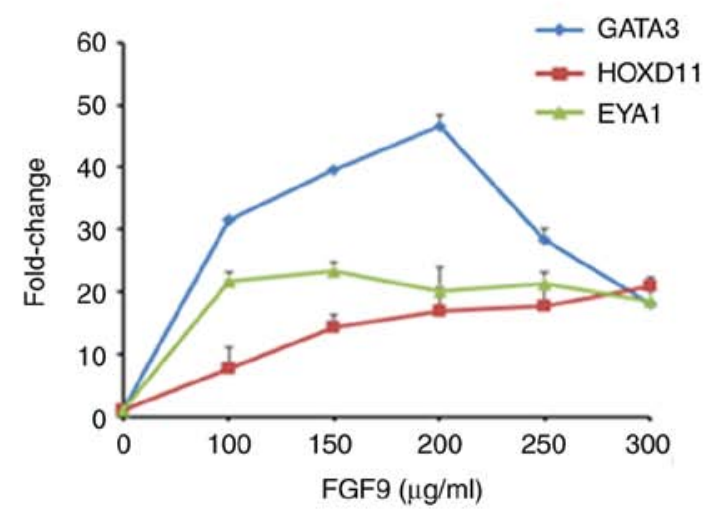

Figure 1. Condition optimization of intermediate mesoderm induced by human induced pluripotent stem cells. (A) Bright field observations at day 4 of induction by gradient concentration of CHIR99021. (B) RT-qPCR detection of the primitive streak markers T, TBX6 and LHX1 at day 4 of induction. (C) RT-qPCR detection of anterior intermediate mesoderm marker GATA3, and posterior intermediate mesoderm markers HOXD11 and EYA1, at day 4 of induction. The data are representative from a minimum of three independent experiments. RT-qPCR, reverse transcription-quantitative PCR; TBX6, T-box transcription factor 6; LHX1, LIM homeobox 1; GATA3, GATA binding protein 3; HOXD11, homeobox 11; EYA1, EYA transcriptional coactivator and phosphatase 1; FGF9, fibroblast growth factor 9.

shape, but the cells gradually exhibited a fuller shape after CHIR99021 was replaced with FGF9 (Fig. 2A). hiPSCs were analyzed and treated with CHIR9901 for 4 days, and immunofluorescence analysis showed that the cells were positive for both T and LHX1 (Fig. 2B). On the 7th day of induction, mesoderm markers were detected, and immunofluorescence analysis demonstrated that the cells were positive for the mesoderm markers GATA3 and WT1 (Fig. 2C).

$3 D$ culture of renal organoids in vitro. The 3D culture of renal organoids in vitro was examined (Fig. 3). The morphology of kidney organoids was viewed under x40 light microscope (Fig. 3A). H\&E staining analyses showed that hiPSCs were induced to form a tubular structure in vitro (Fig. 3B). The expression levels of renal-related molecular markers in the cultured cells were detected by immunofluorescence. The tubule marker LTL was identified to be strongly positive in the tubule structure, while E-cadherin (ECAD) was weakly positive, suggesting that the culture appeared to contain an immature renal tubule structure (Fig. 3C). Some of the tubules were also found to be GATA ${ }^{+} \mathrm{ECAD}^{+}$, which indicated the existence of a collecting tube structure in the culture (Fig. 3D). In addition, the specific markers for ureteric buds, such as RET and WNT11, were detected by western blotting. The expression of WNT11 was highest on the 12th day of induction and then decreased. The expression trend of RET was similar to that of WNT11 (Fig. 3G). These results suggested that the production of ureteric progenitor cells during induction peaks on the 12th day and then decreases. The culture was positive for PODXL, a marker of the renal podocyte, an important cell 
A



D4

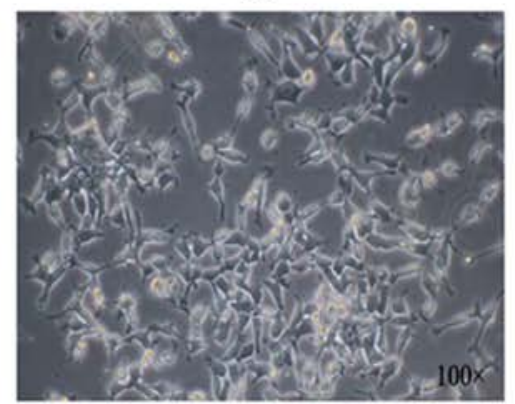

D7

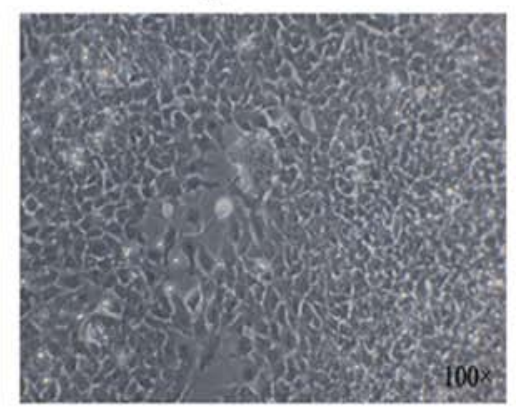

B T DAPI Merged

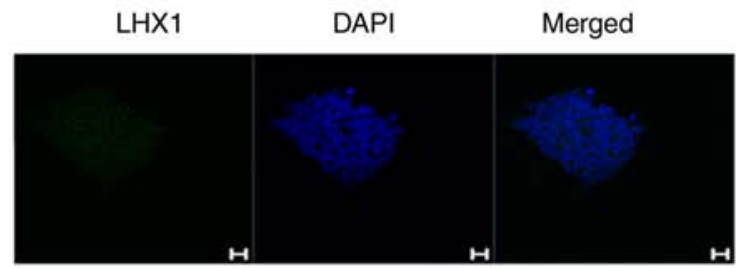

DO
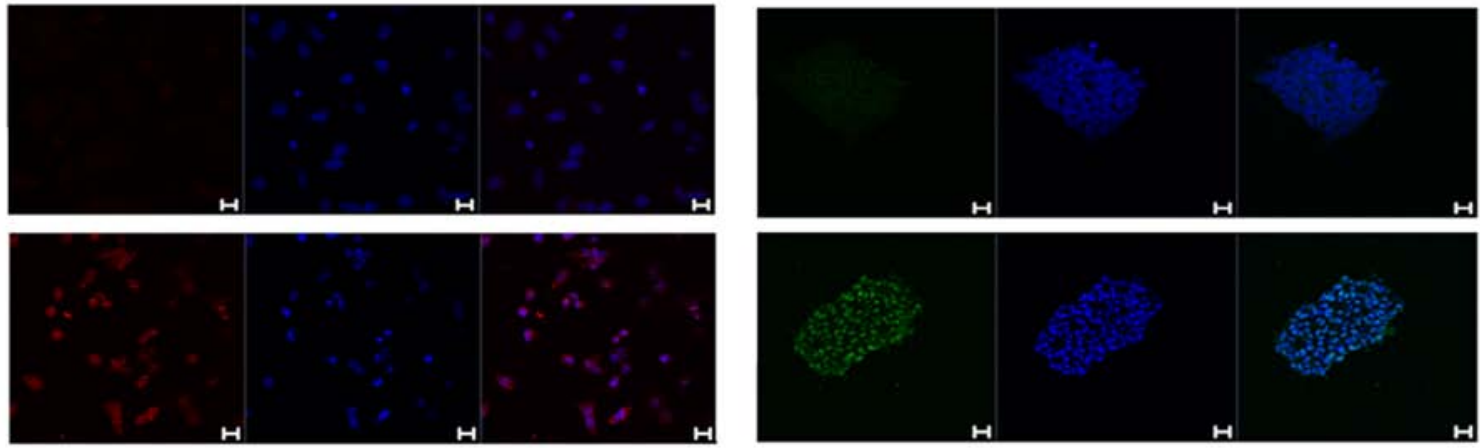

C

GATA3

DAPI

Merged
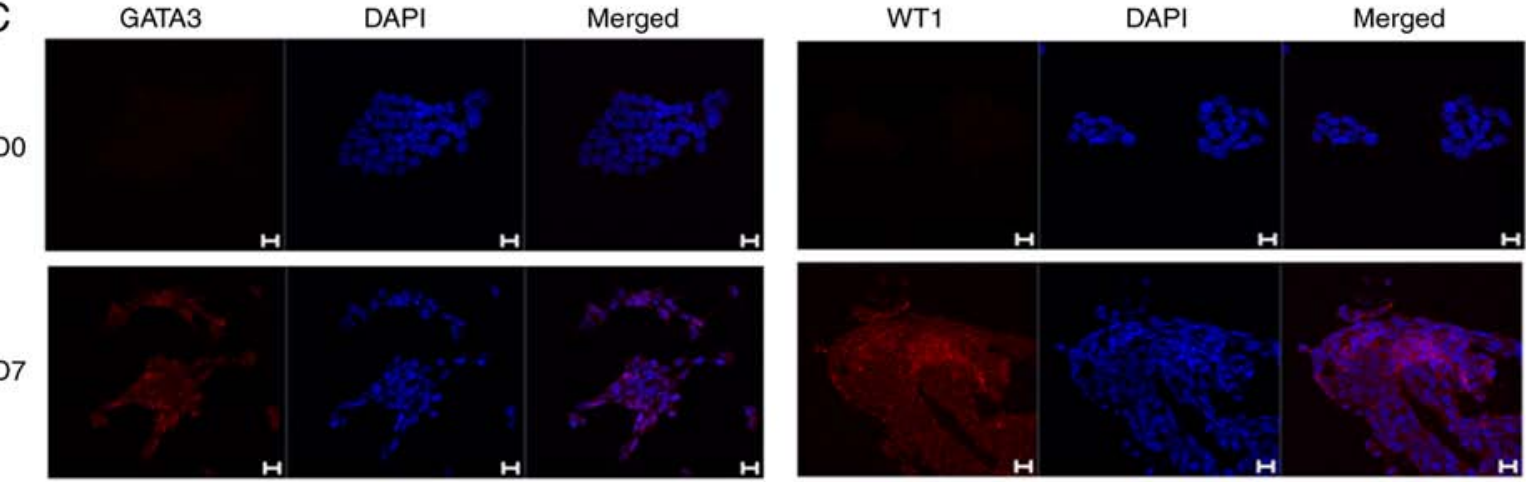

Figure 2. Cell morphology and molecular markers in the mesoderm induction process. (A) Bright field observations during the induction of intermediate mesoderm. (B) Immunofluorescence at day 4 of differentiation with the primitive streak markers, T and LHX1. (C) Immunofluorescence at day 7 of differentiation with the intermediate mesoderm markers, GATA3 and WT1. Scale bars, $20 \mu \mathrm{m}$. The data are representative from a minimum of three independent experiments. D, day; LHX1, LIM homeobox 1; GATA3, GATA binding protein 3; WT1, Wilms tumor protein.

in the glomerular structure (5) (Fig. 3E). To confirm this result, the molecular markers of podocytes (NPHS1, PODXL and WT1) were detected by western blotting (Fig. 3F). WT1 began to be expressed on the 4th day of induction and was still highly expressed on the D18 day of the kidney organoids formation period The other two key podocyte markers were both highly expressed on D18 day of the kidney organoid formation period. These results suggested that renal podocyte-like cells may exist in the kidney organoids.

Renal organoids survive and grow under the kidney capsule. As nutrient supply is a problem during in vitro culture, organoid transplantation was carried out in the present study. The present results demonstrated that the renal capsule provided a differentiation microenvironment that was sufficient for metanephros, while unilateral nephrectomy promoted compensation of the other side of the kidney by increasing the blood supply. On the 12th day of the experiment, the cultured organoids were transplanted into the renal capsules of immunodeficient mice via unilateral nephrectomy (Fig. 4A). After 2 weeks of culture in vivo, the grafts were removed and related molecular markers were detected (Fig. 4B-G). Kidney organoids transplanted in the subcutaneous armpit of mice could not be detected in the control group (data not shown). Statistical analysis identified that the graft volumes (kidney organoids) increased significantly after transplantation, which suggested that the host supplied nutrients for the graft (Fig. 4C). HE staining results showed tubular structures similar to renal tubules (Fig. 4D). A renal tubular marker (LTL) and glomerular marker (NPHS1) were detected using immunofluorescence. The present results demonstrated that some of the grafts were positive for LTL, while the expression 
A

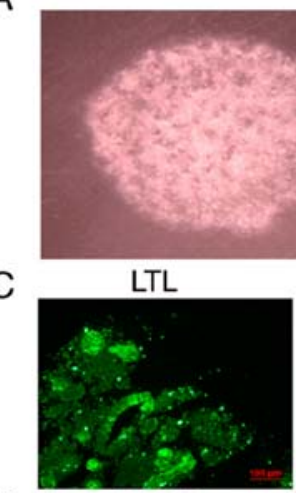

D

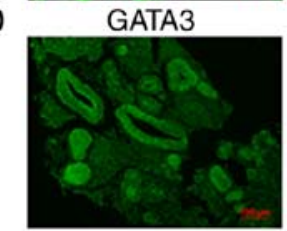

E
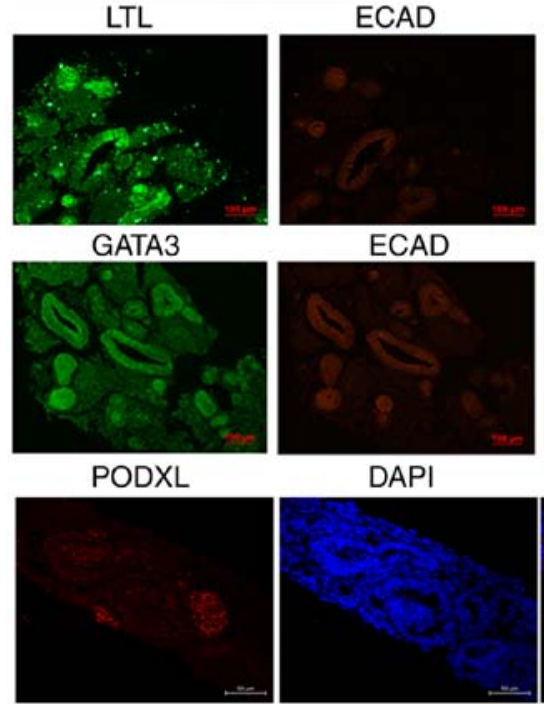

ECAD



DAPI

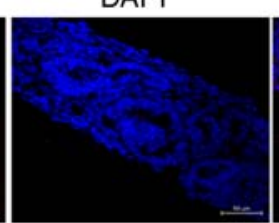

$\mathrm{F}$

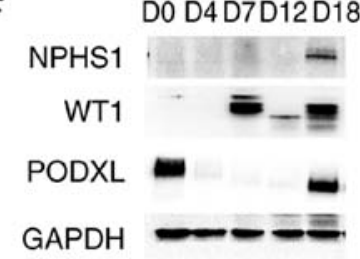

G

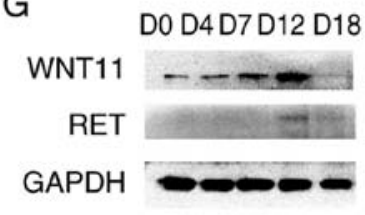

Figure 3. 3D culture of kidney organoids. (A) Bright field observations of the kidney organoids. (B) Hematoxylin and eosin staining results of the kidney organoids. (C) Expression detection of renal tubular markers LTL and ECAD in kidney organoids by immunofluorescence. (D) Expression detection of collection tube markers GATA3 and ECAD in kidney organoids by immunofluorescence. Scale bars, $100 \mu \mathrm{m}$. (E) Expression detection of kidney podocytes marker PODXL in kidney organoids by immunofluorescence. Scale bars, $50 \mu \mathrm{m}$. (F) Expression detection of the molecular markers of podocyte (NPHS1, PODXL and WT1) by western blotting. (G) Expression detection of the specific markers for ureteric buds RET and WNT11 by western blotting. The data are representative from a minimum of three independent experiments. LTL, Lotus tetragonolobus lectin; ECAD, e-cadherin; GATA3, GATA binding protein 3; NPHS1, nephrin; PODXL, podocalyxin; WT1, Wilms tumor protein; RET, proto-oncogene tyrosine-protein kinase receptor Ret; WNT11, protein Wnt-11; D, day.

of ECAD in LTL-positive tubules was negative, indicating that the tubular structure was immature (Fig. 4E). There were sporadic NPHS1-positive grafts, suggesting the presence of kidney podocytes (Fig. 4F). The molecular marker CD31 was partially positive in vascular epithelial cells, indicating the formation of blood cells (Fig 4G).

\section{Discussion}

Organoid-related research has attracted increasing attention, and numerous methods for the in vitro induction of kidney organoids have been reported. Takasato et al (7) induced anterior and posterior intermediate mesoderms by using a Wnt agonist and FGF9 as the main inducers, and these

components were further differentiated into metanephric mesenchymal cells and ureteral progenitor cells, eventually forming kidney organoids. Differentiation of iPSCs is a complex and long-term process. Volpato et al (21) demonstrated that interlaboratory reproducibility of iPSC differentiation is poor and cell type heterogeneity is a major source of interlaboratory variation. Phipson et al (22) analyzed the sources of transcriptional variation in a specific kidney organoid protocol, and demonstrated interexperimental and inter-clonal variation in kidney organoid differentiation within a same laboratory. The interexperimental and inter-clonal variation in kidney organoid differentiation is unavoidable at present. Therefore, at least three biological repeats were required to yield similar results in the present study.

Based on the method established by Taksato et al (7), the conditions for induction at the 2D stage were optimized, demonstrating that $12 \mu \mathrm{M} \mathrm{CHIR} 99021$ led to the most optimal induction when the hiPSCs used in the present study changed to the primitive streak. On the 7th day, RT-qPCR assays indicated that $200 \mathrm{ng} / \mathrm{ml}$ FGF9 led to the best induction, which was consistent with the results of the previous study (7).

The cells were placed on a Transwell membrane and 3D culturing was performed. After 5 days, 200 ng/ml FGF9 was replaced with a simple differentiation medium, APEL, for 2 weeks. Immunofluorescence analysis confirmed that hiPSC-induced kidney organoids in vitro comprised renal tubules, collecting ducts and renal podocytes. However, numerous problems are still associated with inducing kidney organoids in vitro, as renal tubular differentiation is not mature, no mature glomerular structure was observable, and the nutrient supply limitation had not been solved. Another experiment (Fig. S1) and other previous studies $(16,17)$ demonstrated that unilateral nephrectomy promoted the growth of another kidney graft. Kidney organoids induced in vitro were transplanted into the renal capsules of immunodeficient mice and then one of their kidneys was removed to create an environment conducive to kidney organoid growth. At 2 weeks after transplantation, the kidneys were removed, stained with $\mathrm{HE}$ and subjected to immunofluorescence analysis. The present results demonstrated that the kidney organoid volumes were increased significantly compared with those before transplantation, suggesting that the kidney organoids obtained nutrients from the host, and CD31 positivity indicated the presence of vascular endothelial cells (23). However, no positive ECAD expression was observed, which is related to the differentiation and maturation of renal tubules (7). No marked difference in the expression of molecular markers of podocytes was observed between the in vivo and in vitro-induced kidney organoids.

van den Berg et al (13) demonstrated generation of vascularized human kidney tissues derived from hiPSCs upon transplantation of human kidney organoids to renal capsule in mice. In addition, their research demonstrated that renal subcapsular transplantation of PSC-derived kidney organoids induced significant glomerular and tubular maturation in vivo (13). In comparison with the previous study by van den Berg et al (13), the present study has a number of differences, including the animal surgery, in vitro induction time, in vivo culture time and identification methods. The present study demonstrated 


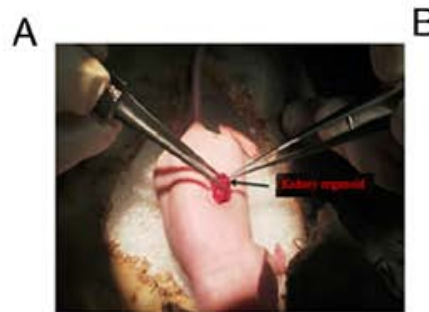

E

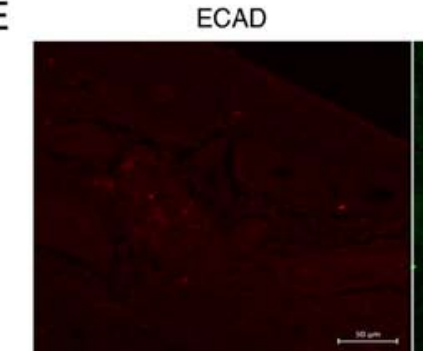

$\mathrm{F}$

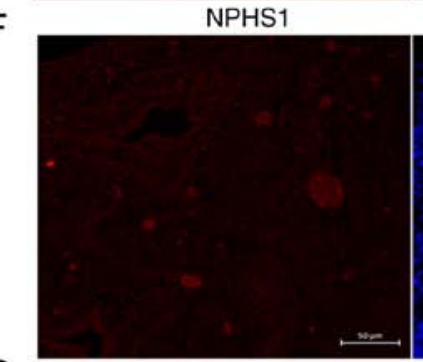

G

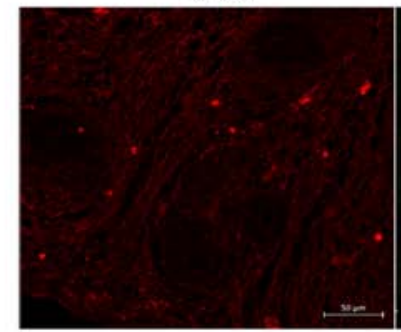

B



LTL

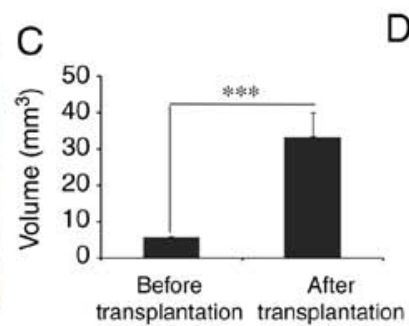

DAPI



Merged
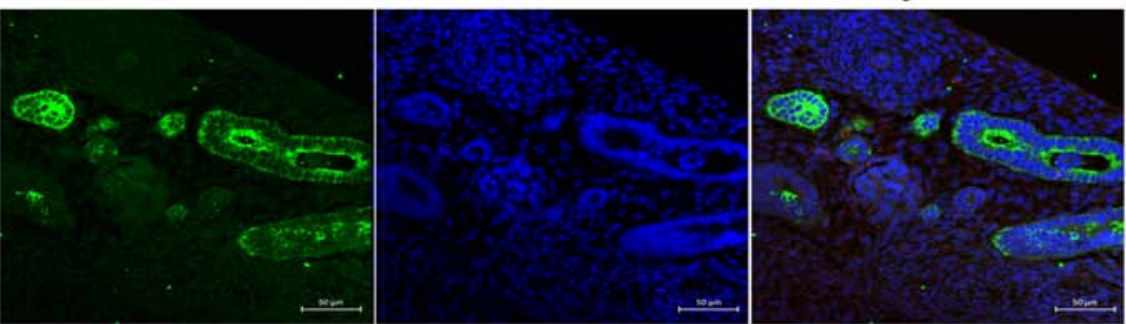

Merged

LTL

DAPI

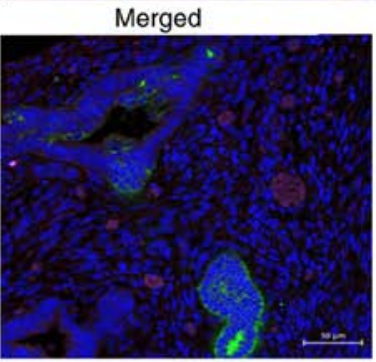

LTL

DAPI

Merged

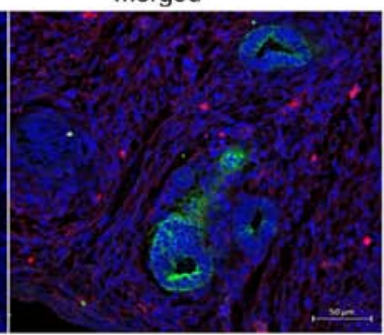

Figure 4. In vivo cultivation and identification of kidney organoids. (A) Kidney organoids were transplanted into the murine renal capsule, and the graft was removed after 2 weeks of culture in vivo. (B) In vivo images two weeks after transplantation. (C) Statistical analysis before and after transplantation. ${ }^{* * *} \mathrm{P}<0.001$. (D) Graft hematoxylin and eosin staining. Scale bar, $100 \mu \mathrm{m}$. (E) Expression detection of renal tubular markers LTL and ECAD in the grafts. (F) Immunofluorescence detection of the molecular marker NPHS1 of renal podocytes in grafts. (G) Immunofluorescence detection of vascular endothelial cell molecular marker CD31. Scale bars, $50 \mu \mathrm{m}$. LTL, Lotus tetragonolobus lectin; ECAD, e-cadherin; NPHS1, nephrin.

that the internal environment can provide nutrients to the graft (kidney organoids), but short-term culture does not significantly promote the differentiation or maturation of renal organ-related cells. Additionally, it was observed that the tubules in the kidney organoids induced or cultured in vitro were very sparse compared with those in the normal kidneys, and a large area of low or osteogenic differentiation was observed, suggesting that the efficiency of kidney organoid induction was still substantially improved. To improve the induction efficiency of kidney organoids, future studies should further optimize the induction conditions, and consider screening renal interstitial cells and ureteral progenitor cells via flow separation, and then culturing these cells. Considering the degree of differentiation observed, the time of kidney organoid culture in vitro or in vivo should be extended. After kidney organoid transplantation, VEGF and FGF9 may be added to promote the angiogenesis and differentiation of renal-related cells.
In summary, the present study demonstrated that the microenvironment of the kidney capsule after unilateral nephrectomy is conducive to the proliferation of kidney organoids. However, there are many limitations in the present study that should be addressed.

\section{Acknowledgements}

The authors would like to thank Dr Yong Guan and Dr Yanxia Guo (Engineering Laboratory of Shandong Province for Structure and Functional Reconstruction of Urinary Organs) for providing helpful discussions and technical support concerning the present study.

\section{Funding}

The present study was supported by the National Natural Science Foundation of China (grant nos. 81700592 and 81670625 ) and the Shandong Provincial Natural Science Foundation (grant no. ZR2017PH058). 


\section{Availability of data and materials}

The datasets used and/or analyzed during the current study are available from the corresponding author on reasonable request.

\section{Authors' contributions}

SZ and FK conducted the study. SZ and GC designed the experiments. DZ, XD, KL and XZ performed experiments, and collected and analyzed the data. All authors read and approved the final manuscript.

\section{Ethics approval and consent to participate}

Animal care and handling were performed in accordance with The National Institutes of Health Guide for the Care and Use of Laboratory Animals. All procedures were approved by The Ethics Committee of The Second Hospital of Shandong University.

\section{Patient consent for publication}

Not applicable.

\section{Competing interests}

The authors declare that they have no competing interests.

\section{References}

1. Ng JK and Li PK: Chronic kidney disease epidemic: How do we deal with it? Nephrology (Carlton) 23: 116-120, 2018.

2. Tsai HB, Chao CT, Chang RE, Hung KY and COGENT Study Group: Conservative management and health-related quality of life in end-stage renal disease: A systematic review. Clin Invest Med 40: E127-E134, 2017.

3. Thuret R, Timsit MO and Kleinclauss F: Chronic kidney disease and kidney transplantation. Prog Urol (French) 26: 882-908, 2016.

4. van Gelder MK, Mihaila SM, Jansen J, Wester M, Verhaar MC Joles JA, Stamatialis D, Masereeuw R and Gerritsen KG: From portable dialysis to a bioengineered kidney. Expert Rev Med Devices 15: 323-336, 2018.

5. Kitamura S, Sakurai H and Makino H: Single adult kidney stem/progenitor cells reconstitute 3-dimensional nephron structures in vitro. Stem Cells 33: 774-784, 2015.

6. Taguchi A, Kaku Y, Ohmori T, Sharmin S, Ogawa M, Sasaki H and Nishinakamura R: Redefining the in vivo origin of metanephric nephron progenitors enables generation of complex kidney structures from pluripotent stem cells. Cell Stem Cell 14: 53-67, 2014.

7. Takasato M, Er PX, Chiu HS, Maier B, Baillie GJ, Ferguson C, Parton RG, Wolvetang EJ, Roost MS, Chuva de Sousa Lopes SM and Little MH: Kidney organoids from human iPS Cells contain multiple lineages and model human nephrogenesis. Nature 526: $564-568,2015$
8. Morizane R, Lam AQ, Freedman BS, Kishi S, Valerius MT and Bonventre JV: Nephron organoids derived from human pluripotent stem cells model kidney development and injury. Nat Biotechnol 33: 1193-1200, 2015.

9. Cunha GR and Baskin L: Use of sub-renal capsule transplantation in developmental biology. Differentiation 9: 4-9, 2016.

10. Schmidt C: Pancreatic islets find a new transplant home in the omentum. Nat Biotechnol 35: 8, 2017.

11. Gould DJ, Mehrara BJ, Neligan P, Cheng MH and Patel KM: Lymph node transplantation for the treatment of lymphedema. J Surg Oncol 118: 736-742, 2018.

12. Hammerman MR: Renal organogenesis from transplanted metanephric primordia. J Am Soc Nephrol 15: 1126-1132, 2004.

13. van den Berg CW, Ritsma L, Avramut MC, Wiersma LE, van den Berg BM, Leuning DG, Lievers E, Koning M, Vanslambrouck JM, Koster AJ, et al: Renal subcapsular transplantation of psc-derived kidney organoids induces neo-vasculogenesis and significant glomerular and tubular maturation in vivo. Stem Cell Rep 10: 751-765, 2018.

14. Urie BK, Tillson DM, Smith CM, Brawner WR, Almond GT, Beard DM, Lenz SD and Lothrop CD Jr: Evaluation of clinical status, renal function, and hematopoietic variables after unilateral nephrectomy in canine kidney donors. J Am Vet Med Assoc 230: 1653-1656, 2007.

15. Kendi Celebi Z, Peker A, Kutlay S, Kocak S, Tuzuner A, Erturk S, Keven K and Sengul S: Effect of unilateral nephrectomy on urinary angiotensinogen levels in living kidney donors: 1 year follow-up study. J Renin Angiotensin Aldosterone Syst 18: $1470320317734082,2017$.

16. Dilworth MR, Clancy MJ, Marshall D, Bravery CA, Brenchley PE and Ashton N: Development and functional capacity of transplanted rat metanephroi. Nephrol Dial Transplant 23: 871-879, 2008.

17. Matsumoto K, Yokoo T, Yokote S, Utsunomiya Y, Ohashi T and Hosoya T: Functional development of a transplanted embryonic kidney: Effect of transplantation site. J Nephrol 25: 50-55, 2012.

18. Wang S, Wang B, Pan N, Fu L, Wang C, Song G, An J, Liu Z, Zhu W, Guan Y, et al: Differentiation of human induced pluripotent stem cells to mature functional Purkinje neurons. Sci Rep 5: 9232, 2015

19. Livak KJ and Schmittgen TD: Analysis of relative gene expression data using real-time quantitative PCR and the 2-Delta Delta C (T)) method. Methods 25: 402-408, 2001.

20. Yuan H, Gong A, and Young CY: Involvement of transcription factor Sp1 in quercetin -mediated inhibitory effect on the androgen receptor in human prostate cancer cells. Carcinogenesis 26: 793-801, 2005.

21. Volpato V, Smith J, Sandor C, Ried JS, Baud A, Handel A, Newey SE, Wessely F, Attar M, Whiteley E, et al: Reproducibility of molecular phenotypes after long-term differentiation to human iPSC-derived neurons: A multi-site omics study. Stem Cell Reports 11: 897-911, 2018.

22. Phipson B, Er PX, Combes AN, Forbes TA, Howden SE, Zappia L, Yen HJ, Lawlor KT, Hale LJ, Sun J, et al: Evaluation of variability in human kidney organoids. Nat Methods 16: 79-87, 2019.

23. Lertkiatmongkol P, Liao D, Mei H, Hu Y and Newman PJ: Endothelial functions of platelet/endothelial cell adhesion molecule-1 (CD31). Curr Opin Hematol 23: 253-259, 2016.

This work is licensed under a Creative Commons Attribution-NonCommercial-NoDerivatives 4.0 International (CC BY-NC-ND 4.0) License. 